LINA MARÍA CÁRDENAS BAYONA

ESCUELA DE DISEÑO

FACULTAD DE ARQUITECTURA, DISEÑO

YESTUDIOS URBANOS

PONTIFICIA UNIVERSIDAD CATÓLICA DE CHILE

SANTIAGO, CHILE

LINA.CARDENAS@UC.CL

\section{Revisión preliminar sobre el control y comunicación de color en el retail textil chileno}

\author{
Preliminary Revision on the Control and Communication of \\ Color in the Chilean Textile Retail
}

Resumen. Este trabajo es el resultado de una revisión preliminar sobre el manejo y gestión del color en la industria de retail chilena, con el objetivo de determinar qué variables son claves dentro de una típica cadena de suministro textil. A partir de entrevistas y encuentras a personas que día a día toman decisiones de color en ese ámbito, se identificaron las variables importantes que podrían influir en el control y gestión de color en el ámbito nacional. Además, se logró identificar que muchas de las prácticas hechas por el retail textil chileno en torno al color, se hacen de una forma intuitiva. La gran mayoría por falta de conocimiento y carencia de una formación en ciencia del color. Está información da las bases para entender cómo funciona la gestión de color a nivel nacional y además permite identificar posibles estrategias para implementar control y comunicación del color en la industria de Retail textil nacional.

Palabras clave: Chile, comunicación de color, gestión de color, retail, textil.

\begin{abstract}
This work is the result of a preliminary revision on the color control and management in the textile retail industry in Chile. This work aimed to determine which variables are key within a typical textile supply chain. Interviews and surveys were carried out among color assessors in the Chilean retail. The most important variables that influence the control and management of color at the national level were identified. In addition, it was determined that many of the practices in color assessment are made very intuitive due to the lack of knowledge and a solid background in color science. This information gives the basis to understand how color management works and it allows to identify possible strategies to implement control and communication of color in the national retail industry.
\end{abstract}

Keywords: Chile, color communication, color management, retail, textile.

Fecha de recepción: 08/05/2017

Fecha de aceptación: 30/06/2017

Cómo citar: Cárdenas Bayona, L. M. (2017).

Revisión preliminar sobre el control y

comunicación del color en el retail textil chileno.

RChD: creación y pensamiento, 2(2), 1-9.

DOI: 10.5354/0719-837X.2017.46101

Revista Chilena de Diseño,

RChD: creación y pensamiento

Universidad de Chile

2017, 2(2)

http://rchd.uchile.cl 


\section{DISEÑO}

\section{PRE-PRODUCCIÓN}

\section{MANUFACTURA}

\section{DISTRIBUCIÓN}

Figura 1. Gráfico del ciclo básico de un producto textil. Fuente: Hinks, Shamey, y Cárdenas (2009).

\section{Introducción}

Cuando se habla de color, no solo se toma el aspecto físico de la luz interactuando con un objeto, sino de la experiencia subjetiva que produce en el ojo y el cerebro de un observador. Desde el punto de vista comercial, el color le da un valor estético a un producto y adicionalmente juega un papel muy importante en la decisión de compra (Butts, 2006). Por lo tanto, la eficiente comunicación de color para todas las industrias en que el color de un producto deba ser controlado para asegurar su consistencia y calidad es fundamental. En los productos textiles, por ejemplo, la interacción entre el diseñador, productor y el punto de venta es crucial para obtener productos de alta calidad y de costo eficiente. La gestión de color en la industria de retail textil, desde el diseño hasta el punto de venta, típicamente incluye el proceso en el que los diseñadores comunican sus requerimientos de color a la cadena de suministro. Usualmente se solicitan muestras físicas (pre-producción) a los proveedores. Una vez aprobado el color, se solicitan muestras de la primera producción antes de empezar la producción en línea; a este proceso le sigue corte y confección, transporte y bodegaje y punto de venta (Little Wood, 2006). La Figura 1 muestra el ciclo básico de desarrollo de un producto textil.

Lo anteriormente descrito, es la versión simplificada de una serie de procesos paralelos que pueden llegar a ser bastante complejos ya que envuelven múltiples actores, barreras de lenguaje, tiempos, etc. (Little Wood, 2006). En Chile, la gestión de color en la industria de retail no es tan diferente en términos generales. Sin embargo, cuando se empieza a analizar la información se advierte cómo hay ciertas prácticas que difieren con lo que se hace a nivel internacional (Fuentes, Müeller, y Vásquez, 2016).

\section{Variables clave en el control de color}

Existen un gran número de variables que pueden generar significativos problemas dentro del control de color. Una ayuda práctica para entender la complejidad de la comunicación de color desde el concepto (diseñador) hasta el punto de venta (consumidor) es el diagrama de espina de pescado o diagrama de causa y efecto. Este diagrama es una herramienta que ayuda a identificar las causas reales o potenciales que contribuyen a un problema (Eckes, 2003; Graystone, 2000). Las causas son organizadas en orden de importancia creando una estructura jerárquica en relación al problema. La variabilidad en el control de color a lo largo de la cadena de suministro textil se puede analizar dividiéndola en cinco grandes categorías:

\footnotetext{
- Concepto

- Factor humano

- Manufactura

- Control de calidad

- Punto de venta
} 


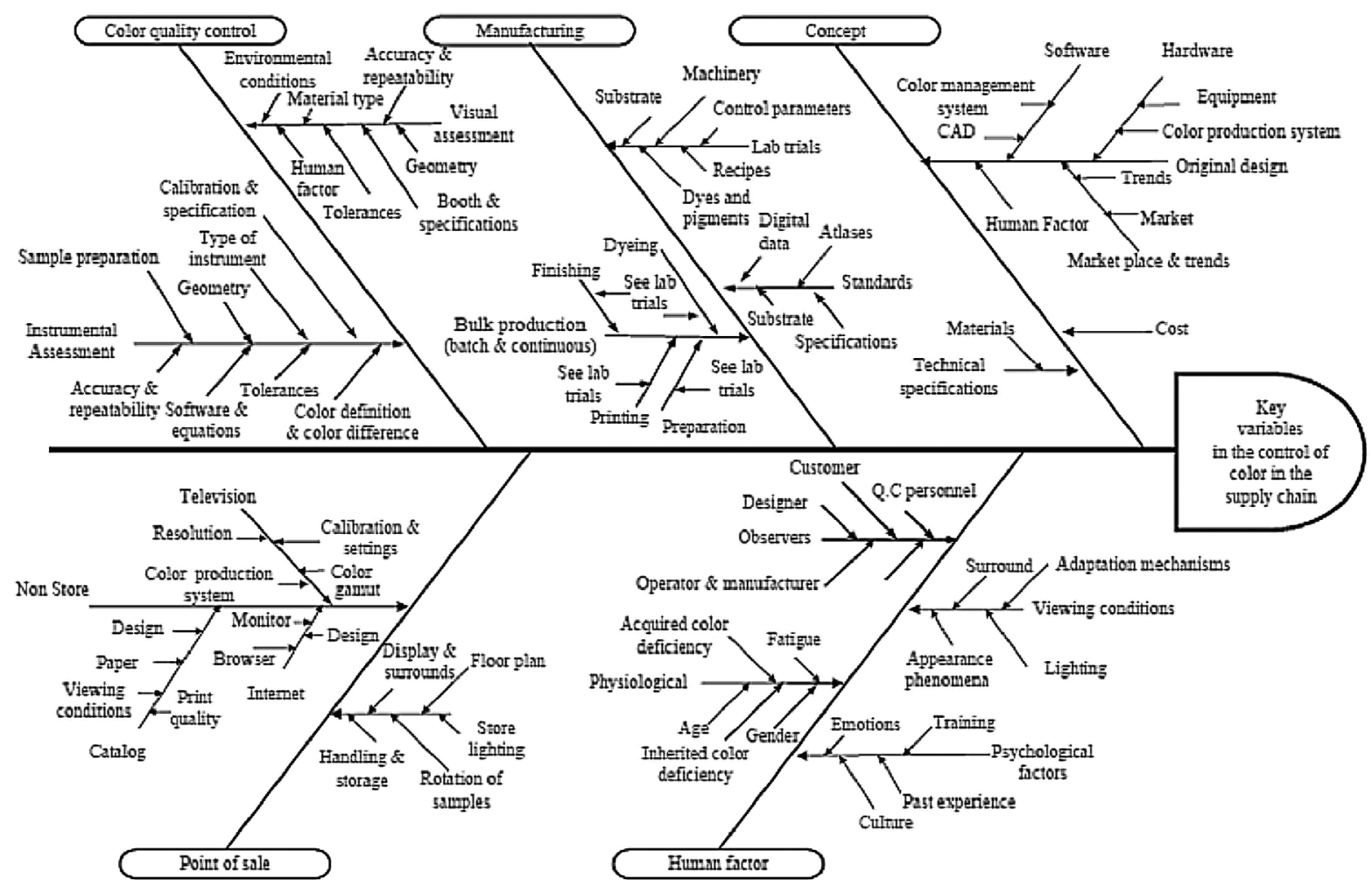

La ilustración de las variables más importantes que causan variabilidad en el control y comunicación de color dentro de la cadena de suministro textil fueron descritas en una publicación anterior (Cárdenas et al., 2006). La Figura 2 muestra la espina de pescado resultante en ese estudio.

Los diagramas de causa y efecto pueden llegar a ser bastante complejos. Sin embargo, el diseño menos complejo presentado anteriormente (Cárdenas et al., 2006), fue usado como marco referencial para comparar y contrastar cada una de las categorías en la industria de retail chilena. La información usada fue obtenida en entrevistas y encuestas a personas que actualmente trabajan en el retail nacional (Fuentes et al., 2016). Si bien la información reportada en este artículo es una primera aproximación a la realidad nacional, los datos demuestran la falta de protocolos en la comunicación de color.

\section{Concepto o diseño original}

Puede decirse con cierta confianza, que un buen diseño es aquel que no solo considera las necesidades del usuario, sino también trabaja con las limitaciones y restricciones de producción.

El color juega un rol vital en casi todas las industrias, especialmente en aquellas donde hay componentes textiles. Una buena elección de color no solo toma en cuenta las necesidades del usuario y las tendencias sino entiende las limitaciones que se puedan presentar en producción (Sanger, 2007). Normalmente, en esta primera etapa, se selecciona cuidadosamente los colores en un diseño. Este proceso implica una cuidadosa planificación y evaluación de los componentes de una colección por parte del equipo de diseño. La selección de colores no solo responde a la tendencia del momento sino también
Figura 2. Diagrama de Causa y Efecto Fuente: Cárdenas et al. (2006). 

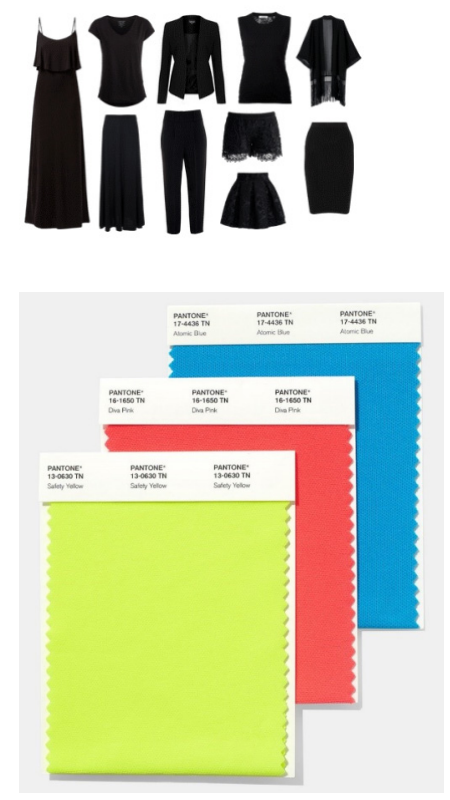

Figura 3. Ejemplo de prendas básicas en un mismo color.

Figura 4. Ejemplo de muestras estándar (xrite). representa la identidad de una marca (Cárdenas et al., 2006). Cada pieza de la colección debe complementar a los demás. En el caso particular chileno, el proceso de diseño comienza con un levantamiento de tendencias a través de agencias externas especializadas en el tema. Aproximadamente cada dos semanas, el grupo de diseño identifica y anticipa qué es lo que viene y cuáles son las tendencias más relevantes para el ámbito nacional (Fuentes et al., 2016). En este proceso también se definen los colores de la colección, los cuales son identificados con la ayuda de la pantonera textil (Cárdenas et al., 2006; Fuentes et al., 2016).

Paralelamente y en conjunto con el área comercial, se eligen las piezas básicas y las cantidades de cada prenda de la colección. Además, se revisan elementos básicos que han funcionado en colecciones anteriores en el tiempo (Fuentes et al., 2016).

Para definir los colores que se van a utilizar, se establece la paleta de color para la colección con la ayuda del Pantone textil (Fuentes et al., 2016) y además se pide una muestra física en textil de los colores seleccionados.

Aunque hoy en día el uso de tecnología para el desarrollo de patrones y prendas de vestir tiene gran auge, la representación del color de diseño "original" en formato digital es muy subjetiva y depende mucho de la calidad del software y de la gama de colores disponibles en el dispositivo en el que se esté trabajando (Cárdenas et al., 2006). En el ámbito nacional, la selección de colores se sigue haciendo visualmente con la ayuda del Pantone textil (Fuentes et al., 2016).

\section{Manufactura}

Reproducir un color en particular de acuerdo a criterios específicos, es uno de los aspectos más desafiantes en el control de color de productos textiles en el retail. A pesar de los avances tecnológicos, la interpretación de un color basado en las inspiraciones conceptuales de un diseñador, no es una tarea fácil (Little Wood, 2006; Sanger, 2007). Dentro del proceso de manufactura, existen claramente dos instancias principales en las cuales se evalúa color. Una primera instancia que tiene como objetivo desarrollar un punto de referencia para la producción en volumen y la segunda producción en masa del producto. En la primera instancia, se debe determinar si los colores enviados por el equipo de diseño son posibles de obtener en el sustrato planeado o hay limitantes técnicas; además, la paleta de color queda desarrollada en una forma más manejable para futuras decisiones (Little Wood, 2006). La industria de retail chilena comunica la paleta desarrollada en códigos Pantone donde además pueden pedir muestras físicas en material textil. Esas muestras son mandadas afuera (principalmente China) en donde se dan especificaciones respecto a que se espera del color. Este proceso es muy iterativo entre el retail y los manufactureros, se requieren envíos constantes de muestras, discusiones, concesiones, pero sobre todo una gran demanda de tiempo y energía. Cabe destacar que este proceso varía mucho en cada compañía, sin embargo, esta parte puede ser muy subjetiva y genera gran variabilidad dado que no existe un protocolo estandarizado. Dentro de la información que se pudo recopilar del ámbito nacional, algunas compañías solo permiten rechazar una muestra de color un máximo de dos veces antes de llevarlo a producción. Otras prefieren omitir un color de la colección cuando desarrollar la muestra es problemático. Algunos retailers solo comunican verbalmente las paletas de 
color y otros trabajan con muestras físicas principalmente en algodón (Fuentes et al., 2016). Sin embargo, en términos de gestión de color, muchas empresas de retail desconocen los procedimientos de las empresas que reproducen color en textiles, generando potenciales problemas en la interacción.

\section{Control de calidad}

Un control preciso de la calidad del color es clave para reducir tiempos y costos en la entrega de productos de color. En la industria textil el control de calidad puede llevarse tanto visual como instrumentalmente. La evaluación visual de color requiere preparación, estandarización y visualización de muestras físicas. Esto inevitablemente implica juicios subjetivos por parte de personal entrenado (American Association of Textile Chemists and Colorists, 2016). Además, el almacenamiento y manipulación de las muestras puede generar un cambio significativo de color, que a su vez puede conducir a potenciales errores en las decisiones de color.

Para evitar la subjetividad que genera la toma de decisiones de color basadas en evaluación visual, el concepto de evaluación instrumental y su aplicación empieza a ser usado en la década de los ochenta en la industria de retail. El uso de tecnología se ha vuelto una herramienta práctica usada en tintura y acabados textiles y en los retails más reconocidos a nivel mundial (Little Wood, 2006). Sin embargo, el uso de medición instrumental de color no es una práctica que en todas las partes del mundo se haya adaptado. En Chile, algunos de retailers hacen evaluación visual usando cabinas estandarizadas de luz.

Las evaluaciones visuales son llevadas a cabo por el equipo de diseño o departamento de compras y las comparaciones son hechas usando el atlas de color Pantone. Adicionalmente, algunos de los retailers cuentan con oficinas en China, donde también se hacen evaluaciones visuales y se aprueba color. Algunos de los retailers nacionales usan como luz estándar la TL84, que es una fuente de luz que simula el iluminante Fil. Esta luz la adoptaron como luz principal para sus evaluaciones visuales, por su amplio uso en el retail europeo, japonés y chino (Fuentes et al., 2016).

Como parte de una investigación sobre las prácticas en gestión de color, 22 encuestas fueron hechas a personas envueltas en toma de decisiones de coIor en la industria de retail textil. La Figura 6 muestra que, a pesar de contar con cabinas para la evaluación de color, 19 de las personas encuestadas no la usan en la toma de decisiones de color.

Igualmente, las encuestas sugieren que no se sigue ningún protocolo de evaluación visual. La Figura 7 muestra la evaluación visual de diferencias de color usando la escala de grises recomendada por la AATCC-American Association of Textile Chemists and Colorists (American Association of Textile Chemists and Colorists, 2016). Dentro de las personas que participaron en las encuestas, tres reconocen usarlas como ayuda en la evaluación visual. (Fuentes et al., 2016)

En Chile, las industrias gráficas, de pinturas y alimentos, han introducido paulatinamente el uso de tecnología en sus prácticas de evaluación de color. Por otra parte, la evaluación instrumental de color no es una práctica adoptada en el retail textil chileno (Sáez, 2016). Muchas de las empresas reconocen no implementarla por un tema de costos y falta de conocimentos en colorimetría (Fuentes et al., 2016; Fuentes, 2016).
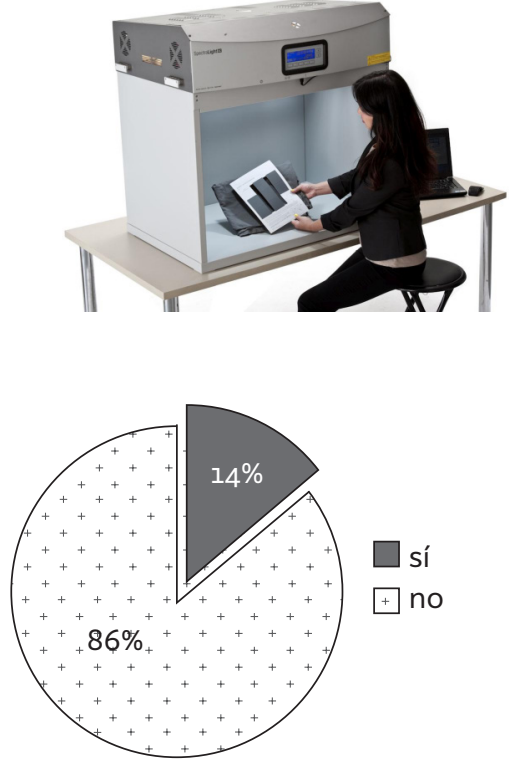

Figura 5. Evaluación visual de color (xrite). Figura 6. Porcentaje uso de cabina de luz. Fuente: Fuentes et al. (2016). 

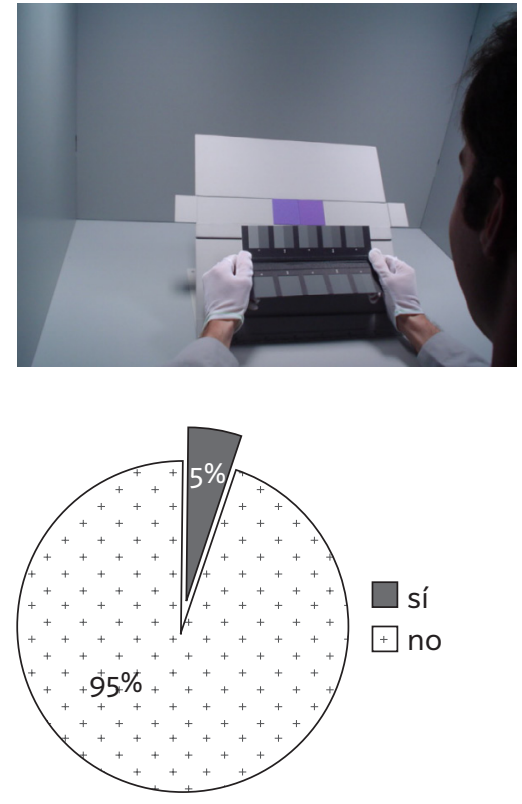

Figura 7. Ejemplo de uso de escala de grises AATCC. Fuente: Cárdenas et al. (2006).

Figura 8. Porcentaje de evaluación instrumental en Chile. Fuente: Fuentes et al. (2016).

\section{Punto de venta}

Las condiciones de visualización de muestras de color, tiene un tremendo impacto en la percepción de productos con color. Existen ciertos fenómenos de percepción como el contraste simultáneo, que afectan la experiencia individual de color de cada persona. El efecto de contraste simultáneo en la percepción de color en textiles está muy bien documentado (Fairchild, 2013; Kuehni, 2012).

La iluminación utilizada para exhibir los productos en el punto de venta de retail, también afecta críticamente el color que se percibe en productos multicomponentes, los que normalmente envuelven cierto grado de inconsistencia de color y metamerismo (Noor, 2004). Cabe destacar que muchas de las importantes cadenas de retail -no solo a nivel nacional sino también internacional-, usan condiciones de iluminación variadas e incontroladas. Asimismo, los diseñadores de visual merchandising suelen cambiar el diseño de la iluminación debido a la temporada u otras decisiones que se necesiten. Estas variaciones pueden resultar en que el color original llegue al punto de venta significativamente diferente, en comparación al diseño original. Es altamente recomendable, que la iluminación que se establezca en el punto de venta físico sea la misma con la que se hacen evaluaciones visuales. Hoy en día el uso de luces LED ha incrementado. El uso de estas lámparas, por parte de las más grandes compañías de retail, está siendo cuidadosamente estudiado, pues son altamente eficientes en consumo de energía además de poder genera luz blanca de una forma económica (Hinks et al., 2009). Adicionalmente, hoy por hoy, el uso de tecnología ha traído otros grandes desafíos para el punto de venta. La proliferación de Smartphones, iPads, ha traído consigo diferentes plataformas para visualizar el color de un producto, lo cual inevitablemente aumenta las posibilidades de variación

\section{El factor humano}

El factor humano es sin duda, uno de los aspectos más importantes dentro del control y comunicación de color en el retail textil. Como se puede ver en el diagrama de espina de pescado anterior, (Cárdenas, Shamey, y Hinks, 2009). Dentro de la cadena de suministro textil hay muchas etapas y procesos que requieren las habilidades visuales de personas. Incluso con procedimientos estandarizados, los seres humanos realizan y hacen evaluaciones diferentes, lo que hace que las decisiones de color sean subjetivas. Estos altos niveles de subjetividad conducen a interpretaciones inconsistentes dentro de la cadena de suministro. Por ejemplo, aunque las propiedades reflectantes de la luz de los objetos puedan ser físicamente cuantificables, el color es una experiencia humana que nunca se puede definir en términos absolutos (Berns, 2000; Kuehni, 2012).

Adicionalmente, la mayoría de los factores que se muestran en la espina de pescado son afectados, en cierta medida, por el factor humano, lo que a su vez provoca variaciones en la evaluación de materiales con color. Algunos de los factores que influyen en las evaluaciones visuales de color incluyen factores psicológicos y ambientales, edad, género, experiencia en el manejo de decisiones relacionadas con color, así como condiciones de visualización (Cárdenas et al., 2009).

Un ejemplo del impacto del factor humano en el control y comunicación de color es la evaluación visual de color. Es de esperarse que todas las personas responsables de las evaluaciones visuales de color sean previamente testeadas 
para probar que tengan visión normal del color (Ishihara, 1936; Nickerson, Granville, y Opt, 1943). (Neitz y Summerfelt, 2001). Sin embargo, dentro de las 22 personas encuestadas que toman decisiones de color en Chile, 13 de ellas no han hecho ningún tipo de test que verifique aquello (Fuentes et al., 2016). Las personas que reconocer haber sido testeadas por su visión de color, también concuerdan que lo hicieron de manera independiente y no como parte del proceso de selección para el cargo. Cabe destacar que las personas encuestadas fueron todas mujeres, lo que es una ventaja, ya la incidencia de problemas de visión de color es menor que en la población masculina (Berns, 2000).

La evaluación del color también está sujeta a la percepción del color de cada individuo, así como de diversos aspectos cognitivos. Los aspectos perceptivos de la evaluación del color incluyen el registro inmediato en el cerebro de los estímulos en términos de luminosidad, tono y croma, mientras que los aspectos cognitivos incluyen el procesamiento posterior de la información como memoria del color, significado del color, etc. (Gao y Xin, 2006). En ese sentido, la experiencia de una persona con visión normal de color es una respuesta del sistema sensorial que es imposible definir. Por otra parte, ciertos aspectos de la psicología del color, como la preferencia de color, pueden influir en la evaluación del color y sesgar la evaluación individual, así como la de grupo. La personalidad, el género, la raza y la edad se han investigado ampliamente como respuesta de grupo en relación a la evaluación de color. Por ejemplo, se ha encontrado que la preferencia y el valor estético de un color está fuertemente influenciado por las diferencias culturales (Whitfield y Whiltshire, 1990). En términos de edad, los resultados de los estudios son ambiguos (Granger, 1955; Staples y Walton, 1933; Whitfield y Whiltshire, 1990).

Además, la optimización de la comunicación en color implica altos niveles de manejo de temáticas de color y conocimientos técnicos expertos en cada etapa de la cadena de suministro. Los resultados de las entrevistas y encuestas muestran que el dominio del color en la industria de retail textil chilena es muy intuitivo. La Figura 9 muestra que únicamente seis de las personas encuestadas recibieron algún tipo de entrenamiento sobre evaluación de color. Adicionalmente, el tema de entrenamiento de color va más allá de la práctica. Un primer acercamiento a la formación de las personas encargadas de tomar decisiones de color demostró que ellos mismos consideran tener un nivel de conocimiento promedio en relación a conceptos básicos de color, limitado a lo aprendido en el colegio (Fuentes et al., 2016)

\section{Conclusiones}

Es importante identificar los parámetros más importantes y las variables que influencian el control de color dentro de las diferentes etapas de la cadena de suministro textil. Para un control efectivo de color, la meta debiese minimizar las fuentes de variación. Inicial y primordialmente, poner atención a la variación en las evaluaciones visuales en materiales textiles. Asimismo, enfatizar en las condiciones en que estas evaluaciones se llevan a cabo durante las etapas de diseño, producción y el punto de venta. Un primer análisis al retail textil chileno pudo lograrse con la ayuda del diagrama de espina de pescado usado anteriormente.

Las entrevistas y encuestas hechas a un grupo de personas que directamente están involucradas en la toma de decisiones en el retail textil chileno indican que hay poco control de las variables existentes. La mayor parte del esfuerzo
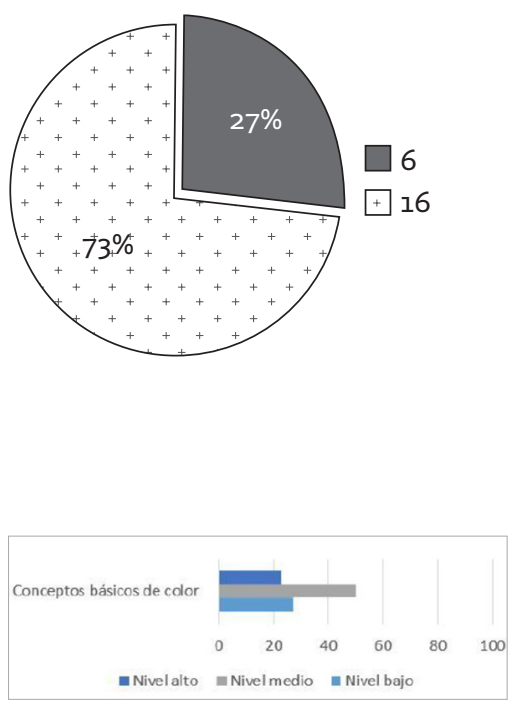

Figura 9. Porcentaje de entrenamiento en evaluación visual de color. Fuente: Fuentes et al. (2016). Figura 10. Nivel de conocimiento en color. 
por parte de las compañías, se centra más en establecer un color que marque tendencia. La optimización de las variables que influyen en la evaluación de color es vital sobre todo para reducir tiempos y mejorar la competitividad. En relación a los protocolos y las prácticas de las evaluaciones de color, la información obtenida en las encuestas y entrevistas sugiere que principalmente predomina el control visual de color entre las empresas de retail textil en Chile, y aunque existan cabinas de luz estandarizadas para la evaluación del color, el personal no las usa regularmente.

Asimismo, se reconoce que hay una falta de conocimiento sobre el color y cómo este puede repercutir en diferentes etapas de la cadena. Por ejemplo, no es requisito tener visión normal de color, aunque el personal esté encargado de tomar decisiones en torno a él. Existe también un porcentaje muy bajo de personas que consideran tienen una buena formación y entrenamiento en la toma de decisiones de color. El nivel de conocimiento sobre color se queda fundamentalmente limitado a la formación que ha recibido cada empleado durante su proceso educativo, que en general no supera el nivel de escolaridad media.

\section{Agradecimientos}

El autor quiere agradecer especialmente a Daniela Fuentes, Macarena Müller y Camilo Vásquez por su valiosa colaboración haciendo las entrevistas y encuestas. 


\section{Referencias}

American Association of Textile Chemists and Colorists. (2016). Evaluation procedure 9. AATTCC technical manual (pp. 435-437). New York: AATTcC

Berns, R. S. (2000). Billmeyer and Saltzman's principles of color technology. New York: John Wiley \& Sons.

Butts, K. (2006). 5 - A practical guide to visual evaluation of textile samples. En: J. H. Xin (Ed.), Total colour management in textiles (pp. 76-93). Cambridge, England: Woodhead Publishing.

Cárdenas, L., Hinks, D., Shamey, R., Kuehni, R., Jasper, W., y Gunay, M. (2006). Comparison of naïve and expert observers in the assessment of small color differences between textile samples. Conference on Colour in Graphics, Imaging, and Vision, 2006(1), 341-344.

Cárdenas, L. M., Shamey, R., y Hinks, D. (2009). Key variables in the control of color in the textile supply chain. International Journal of Clothing Science and Technology, 21(5), 256-269.

Eckes, G. (2003). Six sigma for everyone. New York: John Wiley \& Sons.

Fairchild, M. D. (2013). Color appearance models. New York: John Wiley \& Sons.

Farnsworth, D. (1943). The Farnsworth-Munsell 100-hue and dichotomous tests for color vision. JOSA, 33(10), 568-578.

Fuentes, D., Müeller, M., y Vásquez, C. (2016). Control de calidad de color en Chile: ¿Pérdida o ganancia en la industria retail textil? Manuscrito no publicado.

Fuentes, E. (2016). El Retail en Chile: Evaluación instrumental. Entrevista de L. Cárdenas, Santiago, Chile.

Gao, X., y Xin, J. H. (2006). Investigation of human's emotional responses on colors. Color Research \& Application, 31(5), 411-417.

Granger, G. (1955). An experimental study of colour preferences. The Journal of General Psychology, 52(1), 3-20.

Graystone, J. (2000). Integrating colour delivery skills. Ponencia, PRA Conference on the Colour Delivery Challenge, Leeds University, Leeds.

Hinks, D., Shamey, R., y Cárdenas, L. (2009). Digital color management in the textile supply chain. Manuscrito no publicado.

Ishihara, S. (1936). Series of plates designed as tests for colour-blindness.

Kuehni, R. G. (2012). Color: An introduction to practice and principles. New York: John Wiley \& Sons.

Little Wood, G. (2006). 7 - Effective colour communication from mind to market. En: J. H. Xin (Ed.), Total colour management in textiles (pp. 117-135). Cambridge, England: Woodhead Publishing.
Neitz, J., y Summerfelt, P. (2001). The Neitz Test of Color Vision. Los Angeles, CA: Western Psychological Service. Noor, K. (2004). Effect of lighting variability on the color difference assessment. North Carolina State University. Recuperado de http://www.lib.ncsu.edu/resolver/1840.16/262

Sáez, P. (2016). El Retail en Chile: Evaluación instrumental. Entrevista de L. Cárdenas, Santiago, Chile.

Sanger, A. (2007). Creativity: Getting color right. Color Management Workshop, Raleigh, NC.

Staples, R., y Walton, W. E. (1933). A study of pleasurable experience as a factor in color preference. The Pedagogical Seminary and Journal of Genetic Psychology, 43(1), 217-223.

Whitfield, T., y Whiltshire, T. (1990). Color psychology: A critical review. Genetic, Social, and General Psychology Monographs, (116), 387-412. 